\title{
Temperature-Dependent SRS Behavior of 316L and Its Constitutive Model
}

\author{
Jian Peng ${ }^{1,2} \cdot$ Jian Peng ${ }^{1} \cdot$ Kai-Shang $\mathrm{Li}^{1} \cdot$ Jun-Feng Pei ${ }^{1} \cdot$ Chang-Yu Zhou ${ }^{3}$ \\ Received: 28 October 2017/Revised: 28 November 2017/Published online: 3 January 2018 \\ (C) The Chinese Society for Metals and Springer-Verlag GmbH Germany, part of Springer Nature 2018
}

\begin{abstract}
The strain rate sensitivity (SRS) and temperature sensitivity (TS) of 316L austenitic stainless steel were investigated by constant strain rate test (CSRT) and strain rate jump test (SRJT) under four temperatures $(293,373,473$ and $573 \mathrm{~K})$ and four strain rates $\left(5 \times 10^{-4} / \mathrm{s}, 1 \times 10^{-3} / \mathrm{s}, 5 \times 10^{-3} / \mathrm{s}\right.$ and $\left.1 \times 10^{-2} / \mathrm{s}\right)$. The results show that temperature sensitivity (TS) indexes at different strain rates are coincidence to be negative, related to temperature softening. On the contrary, SRS indexes change from positive to negative with the increase in temperature associated with dynamic strain aging (DSA). Moreover, based on the comparison between CSRT and SRJT, SRS and TS indexes obtained by two methods agree well. It proves that the SRJT can describe the SRS and TS phenomenon of 316L efficiently. Furthermore, the effects of temperature and strain rate on fracture mechanism were discussed. At last, an improved Johnson-Cook model was proposed to consider the temperature-dependent SRS behavior of 316L.
\end{abstract}

Keywords Strain rate sensitivity $\cdot$ Strain rate jump test $\cdot$ Improved Johnson-Cook model

\section{Introduction}

Generally, strain rate and temperature play important roles in tensile behaviors of various metals, and strain rate sensitivity (SRS) was paid attention for decades. Lots of metals display strain rate hardening behavior, such as $\mathrm{Ni}-$ based super alloy [1], magnesium alloy [2], CP-Ti [3] and stainless steel [4]. Differing from the strain rate hardening, negative strain rate sensitivity induced by dynamic strain aging (DSA) was detected for some metals, such as $\mathrm{Fe}-$ 22Mn-0.6C [5], dual-phase steel [6] and twinning-induced plasticity steel [7]. In previous studies of SRS behavior, the constant strain rate test (CSRT) is the most widely used method. Recently, the strain rate jump test (SRJT) was

Available online at http://link.springer.com/journal/40195

Jian Peng

joepengjian@163.com

1 School of Mechanical Engineering, Changzhou University, Changzhou 213164, China

2 Jiangsu Key Laboratory of Green Process Equipment, Changzhou University, Changzhou 213164, China

3 School of Mechanical and Power Engineering, Nanjing Tech University, Nanjing 210009, China utilized to the studies of SRS for austenitic steel [8]. The difference between two methods is the control mode of strain rate. Comparing with classical CSRT, the SRJT can decrease the consumed time during experiments and the discreteness among different specimens. However, the entire stress-strain curve at a specified strain rate and temperature can hardly be realized by SRJT. Therefore, the rationality of SRJT needs to be further proved, especially for the determination of SRS and TS by this test method.

Constitutive model is generally introduced to quantify the mechanical behavior of metallic material. Arrhenius [9], Johnson-Cook [10,11] and Zerrili-Armstrong [12] are commonly used to describe the relationship of stress-strain curve with strain rate and temperature. In addition, artificial neural network can also be put into the application to predict the stress-strain curves at different strain rates and temperatures, which is built on artificial intelligence technology [13]. Lots of studies took efforts to develop constitutive models, especially for $\mathrm{J}-\mathrm{C}$ model $[14,15]$. Lin et al. [16] improved $\mathrm{J}-\mathrm{C}$ model by considering the coupling effects of temperature and strain rate for high-strength alloy steel. Li et al. [17] used the improved $\mathrm{J}-\mathrm{C}$ model proposed by Lin to predict the hot deformation behavior of $28 \mathrm{CrMnMoV}$ steel with suitable accuracy. Since strain hardening (SH), SRS and TS are independently considered 
in $\mathrm{J}-\mathrm{C}$ model, the classical $\mathrm{J}-\mathrm{C}$ model needs to be developed for the temperature-dependent SRS behavior.

Due to the merits of austenitic stainless steel such as corrosion resistance and excellent welding performance [18-20], it was widely applied in engineering equipment. In order to further understand the tensile behavior of austenitic stainless steel, both CSRT and SRJT are utilized to analyze SRS and TS, especially for the temperature-dependent SRS. And results of SRJT and CSRT are compared to verify the effectiveness of SRJT. Moreover, the effects of temperature and strain rate on fracture mechanism are discussed. At last, based on the SRS and TS of 316L, the constitutive model is constructed and improved for reasonably describing the tensile behavior of $316 \mathrm{~L}$ at room and intermediate temperatures. Better understanding the mechanical behavior of $316 \mathrm{~L}$ and its constitutive model can give the theoretical basis for its engineering application.

\section{Experiment and Results}

\subsection{Tensile Experiments}

In this study, 316L tensile specimens were manufactured on the basis of tensile testing standards [21] and were processed by wire cutting to reduce the size deviation. Furthermore, the same roughness was achieved by polishing the specimen surface with 1500 grain grades of abrasive paper. Tensile experiments were performed on the universal testing machine. For the sake of examining the TS of $316 \mathrm{~L}$, different temperatures $(293,373,473$ and $573 \mathrm{~K})$ were considered. The temperature-controlled cabinet and thermocouple were used to control and monitor temperature during tensile tests. In order to examine the SRS of $316 \mathrm{~L}$, different strain rate levels $\left(5 \times 10^{-4} / \mathrm{s}\right.$, $1 \times 10^{-3} / \mathrm{s}, 5 \times 10^{-3} / \mathrm{s}$ and $1 \times 10^{-2} / \mathrm{s}$ ) were considered at different temperatures. The change of strain rate was realized by controlling the displacement rate of machine chuck, and the relationship between displacement rate and initial strain rate is $v=\dot{\varepsilon} l$, where $v(\mathrm{~mm} / \mathrm{s})$ is the displacement rate, $\dot{\varepsilon}\left(\mathrm{s}^{-1}\right)$ is the initial strain rate and $l(\mathrm{~mm})$ is the initial gage length of specimen. In this study, the initial strain rate is considered, which agrees with lots of studies $[1,3]$.

Both CSRT and SRJT were conducted in this study. Figure 1a illustrates the comparison between CSRT and SRJT in the control mode of strain rate. For CSRT as a conventional tensile test, the temperature and strain rate of each specimen keep invariant during the testing process until the specimen is fractured. In contrast, for SRJT strain rate increases step by step at some strain values.

\subsection{Results of SRJT and CSRT}

Figure 1b shows the comparison of stress-strain curves obtained by CSRT and SRJT for $316 \mathrm{~L}$ at different temperatures and tensile strain rates. The strength of $316 \mathrm{~L}$ decreases with the increase in temperature, which indicates a significant temperature softening phenomenon. At the same time, the variation of tensile strength with strain rate shows less significance. To clearly display the SRS of $316 \mathrm{~L}$, the magnified views are represented in Fig. 1c-f. According to the results at four temperatures, the stressstrain curves of CSRT and SRJT agree well, and some interesting phenomena can be drawn. As shown in Fig. 1c, $\mathrm{d}$, when the selected temperatures are 293 and $373 \mathrm{~K}$, the relationship between flow stress and strain rate shows a positive dependence and indicates the positive SRS index. However, when the temperature increases to $573 \mathrm{~K}$ as shown in Fig. 1f, the flow stress decreases gradually with strain rate showing a negative SRS index. From the analyses above, the SRS index varies from a positive value to a negative value with the increase in temperature.

\section{Discussion}

Figure 1 illustrates the variation of stress-strain curves with strain rate and temperature, and the variation of SRS with temperature is analyzed in brief. In order to further study SRS and TS of 316L clearly, the quantitative analyses of SRS index and TS index are necessary.

\subsection{SRS by CSRT}

Based on the CSRT method, Backofen et al. [22] proposed an empirical formula of SRS index for plastic materials:

$\sigma=K \dot{\varepsilon}^{\lambda}$,

where $K$ is material parameter, $\sigma(\mathrm{MPa})$ is flow stress, $\dot{\varepsilon}$ $\left(\mathrm{s}^{-1}\right)$ is strain rate, and $\lambda$ is the SRS index. Taking Eq. (1) to the logarithm form:

$\ln \sigma=\ln K+\lambda \ln \dot{\varepsilon}$.

It can be found that the SRS index $\lambda$ is the slope of $\sigma$ and $\dot{\varepsilon}$ in logarithmic coordinate, as Eq. (3).

$\lambda=\mathrm{d} \ln \sigma / \mathrm{d} \ln \dot{\varepsilon}$.

The relationships between $\sigma$ and $\dot{\varepsilon}$ in logarithmic coordinate at different temperatures can be acquired by experimental data. Meanwhile, as these points displayed in Fig. 2, the corresponding SRS indexes at different temperatures and strains are summarized. The SRS index decreases with increasing strain at all temperatures. Moreover, the temperature has a great effect on the SRS 

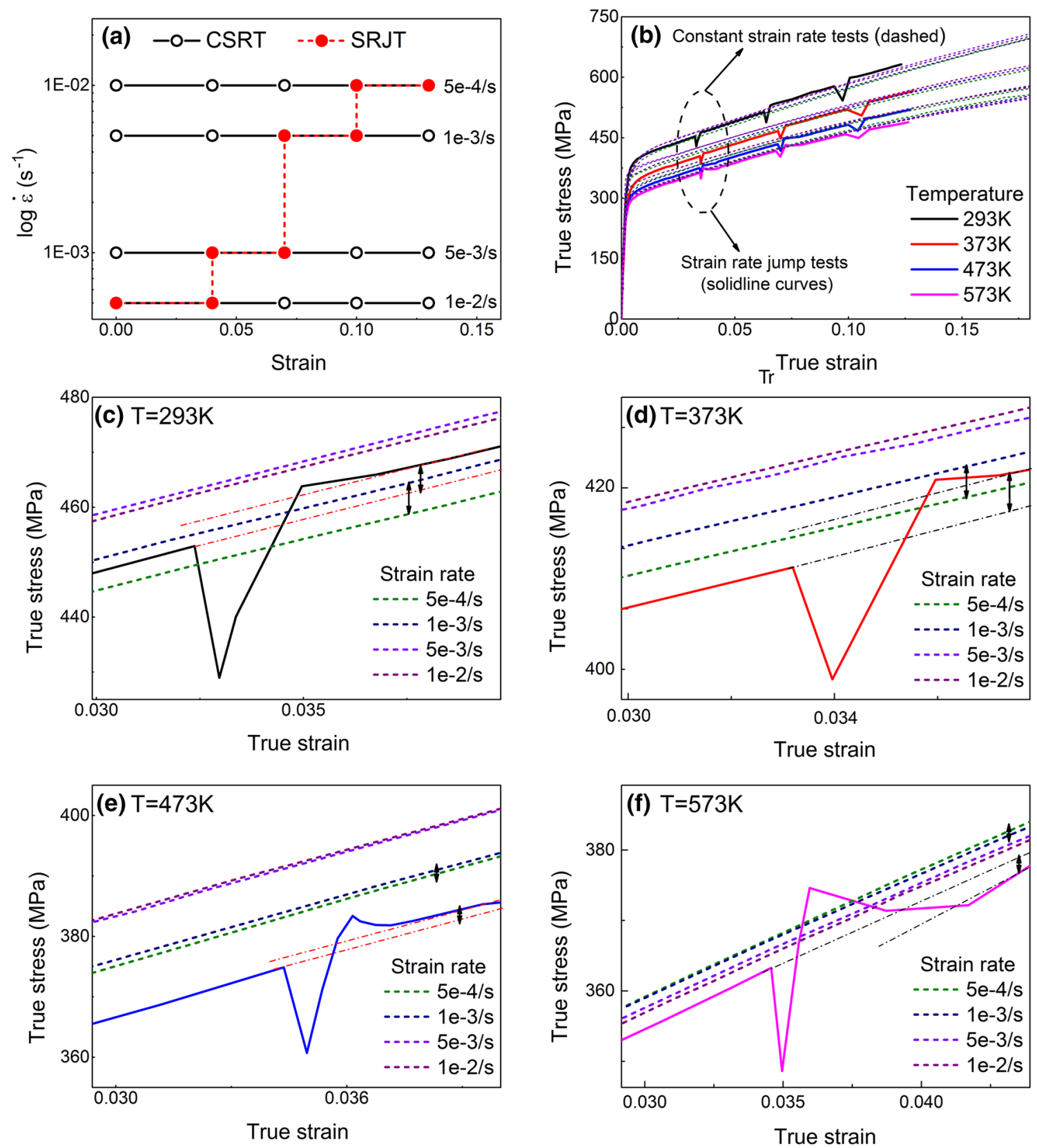

Fig. 1 Comparison between the results of SRJT and CSRT: a the control mode of strain rate; $\mathbf{b}$ the entire view of stress-strain curves; $\mathbf{c}-\mathbf{f}$ the local magnified views at different temperatures

index for 316L steel. At $293 \mathrm{~K}$ (room temperature) and $373 \mathrm{~K}$, the SRS indexes display positive values, showing the strain rate hardening phenomenon. At $473 \mathrm{~K}$, the SRS indexes are mainly positive, but the values are gradually closed to zero with the increase in strain. At $573 \mathrm{~K}$, the SRS indexes are all negative at all strains. The negative SRS indexes at $573 \mathrm{~K}$ for $316 \mathrm{~L}$ are related to the appearance of DSA. One of the macrocharacters of DSA is the negative SRS index [23]. In the previous studies of DSA for 316LN [24], the negative SRS index caused by DSA at the intermediate temperature was found, which is consistent with the results in Fig. 2 in this study. On the basis of the above analyses, the SRS index changes from positive to negative with the increase in the temperature, which gives the evidence that the SRS index is temperature dependent.

\subsection{SRS by SRJT}

Since the SRJT can hardly obtain the entire stress-strain curves at different strain rates, the approach to calculate the SRS index needs to be updated for this experimental 


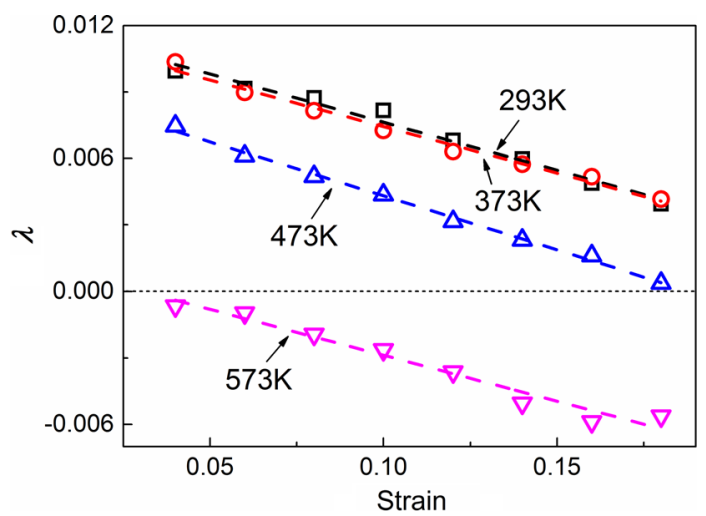

Fig. 2 SRS indexes obtained by CSRT at different temperatures

method. In this study, the back extrapolation method [25] is adopted to calculate the SRS index of SRJT. As shown in Fig. 3, the application of this method to the results of $316 \mathrm{~L}$ at $293 \mathrm{~K}$ is depicted as an example. From Fig. 3a, the stress-strain curve corresponding to the SRJT is given, and the enlarged section of two adjacent strain rates is plotted in Fig. 3b. When the strain rate jumps from $1 \times 10^{-3} / \mathrm{s}$ to $5 \times 10^{-3} / \mathrm{s}$, the intersection points between two extension lines corresponding to two strain rates and the vertical line of the changing strain point are set as $\sigma_{1}(\mathrm{MPa})$ and $\sigma_{2}$ (MPa), respectively. Equation (4) shows the calculation formula of SRS index for SRJT.

$\lambda=\left(\ln \sigma_{2}-\ln \sigma_{1}\right) /\left(\ln \dot{\varepsilon}_{2}-\ln \dot{\varepsilon}_{1}\right)$.

Based on this formula, Fig. 4 shows SRS indexes obtained by SRJT at different temperatures and different strains. As shown in Fig. 4, SRS indexes of SRJT are positive at 293,373 and $473 \mathrm{~K}$. As the temperature is up to $573 \mathrm{~K}$, SRS indexes by SRJT are negative at all strains. Therefore, SRS indexes of SRJT can also illustrate the variation of SRS index with temperature. The temperaturedependent SRS on the basis of SRJT also gives the evidence that the $316 \mathrm{~L}$ tensile behavior is changing from

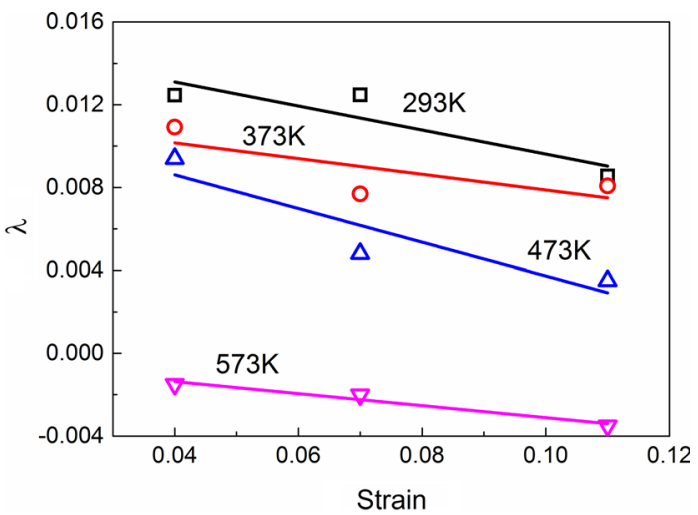

Fig. 4 SRS indexes obtained by SRJT at different temperatures

strain rate hardening to dynamic strain aging with the increase in temperature.

\subsection{TS by CSRT and SRJT}

According to the results of both CSRT and SRJT as shown in Fig. 1b, the TS phenomenon appears. Inspired by the SRS empirical equation, the calculation of TS index is introduced to quantitatively analyze the TS phenomenon in this study as follows:

$\sigma=K T^{\theta}$,

where $K$ is the material constant parameter, $\sigma(\mathrm{MPa})$ is the flow stress, $T(\mathrm{~K})$ is the experimental temperature and $\theta$ is the TS index.

The derivation process of Eq. (6) is similar to that of Eq. (3).

$\theta=\mathrm{d} \ln \sigma / \mathrm{d} \ln T$.

Figure 5a displays the curves of flow stress and temperature in logarithmic coordinate at strain rates $=5 \times 10^{-4} / \mathrm{s}$ by CSRT. According to the slopes of stress and temperature in logarithmic coordinate, the TS
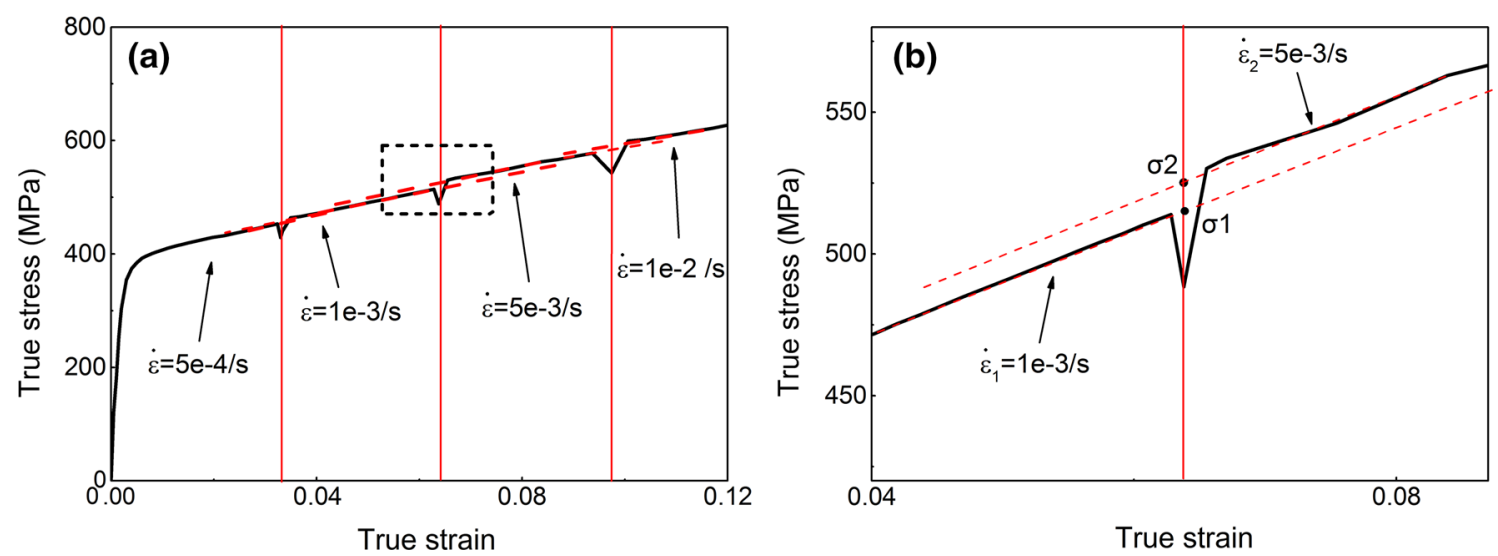

Fig. 3 Back extrapolation method to calculate the SRS index: a the entire view; $\mathbf{b}$ the local enlarged section 

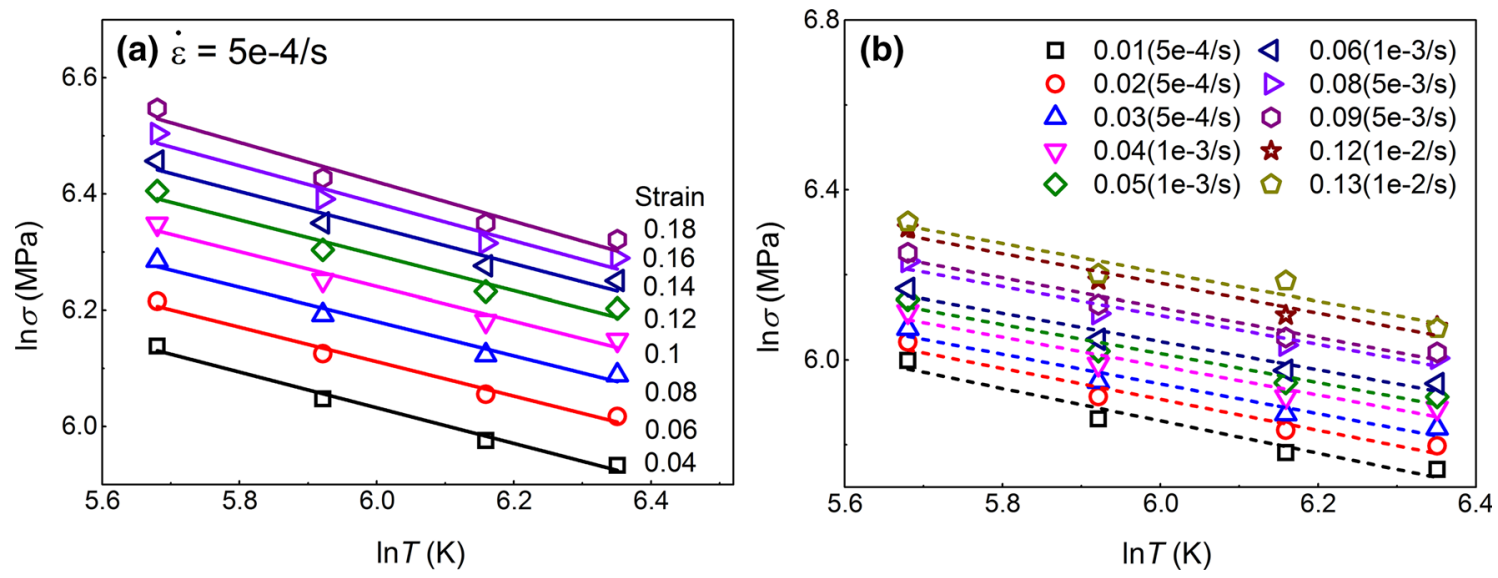

Fig. 5 TS indexes at different strains and strain rates: a the results of CSRT (strain rate $=5 \times 10^{-4} / \mathrm{s}$ ); $\mathbf{b}$ the results of SRJT

indexes can be calculated. The fitting curves at different strains and strain rates are generally parallel, which indicates that TS indexes at full strain and strain rate range have little difference. As shown in Fig. 5b, the curves of flow stress and temperature are given in logarithmic coordinate at different strains and strain rates by SRJT. The slopes of different strains and strain rates are similar. Based on the analyses of the results of both CSRT and SRJT, TS indexes at different strain rates have little difference.

\subsection{Comparison Between SRJT and CSRT}

In order to analyze the differences of the results obtained by two methods, Fig. 6a compares the SRS indexes of SRJT with those of CSRT. From Fig. 6a, the variation of 316L tensile behavior from strain rate hardening to strain rate softening can be found by both methods, which indicates the temperature dependence of SRS index. Figure $6 \mathrm{~b}$ compares the TS indexes of SRJT with those of CSRT. It is worth noting that TS indexes obtained by both methods agree well, and they both have little dependence of strain and strain rate. The results of Fig. 6 illustrate that the TS index and the SRS index obtained by SRJT are effective. Therefore, the SRJT testing method is a proper choice to study the SRS and TS of 316L tensile behavior.

\subsection{Analysis of Fracture Morphology}

For the sake of analyzing the influence of temperature on fracture morphology of $316 \mathrm{~L}$ tensile behavior, the fracture morphologies at different temperatures are displayed in Fig. 7 with the strain rate of $5 \times 10^{-4} / \mathrm{s}$. The high density of dimples can be found at full temperature range as shown in Fig. 7a, c, e, g, which indicates that the failure mechanism at the considered temperature range is ductility controlled. But fracture morphologies at different temperatures give some differences. On one hand, dimples are enlarging and deepening with the increase in temperature. At $293 \mathrm{~K}$ as shown in Fig. 7a, the fracture surface is full of plane and small dimples, but at $573 \mathrm{~K}$ as shown in Fig. $7 \mathrm{~g}$, several large size and deep dimples appear. The reason is that, with the increase in temperature, small dimples will coalesce, and the size of dimple will enlarge with temperature. In the study of the temperature-dependent fracture mechanism for
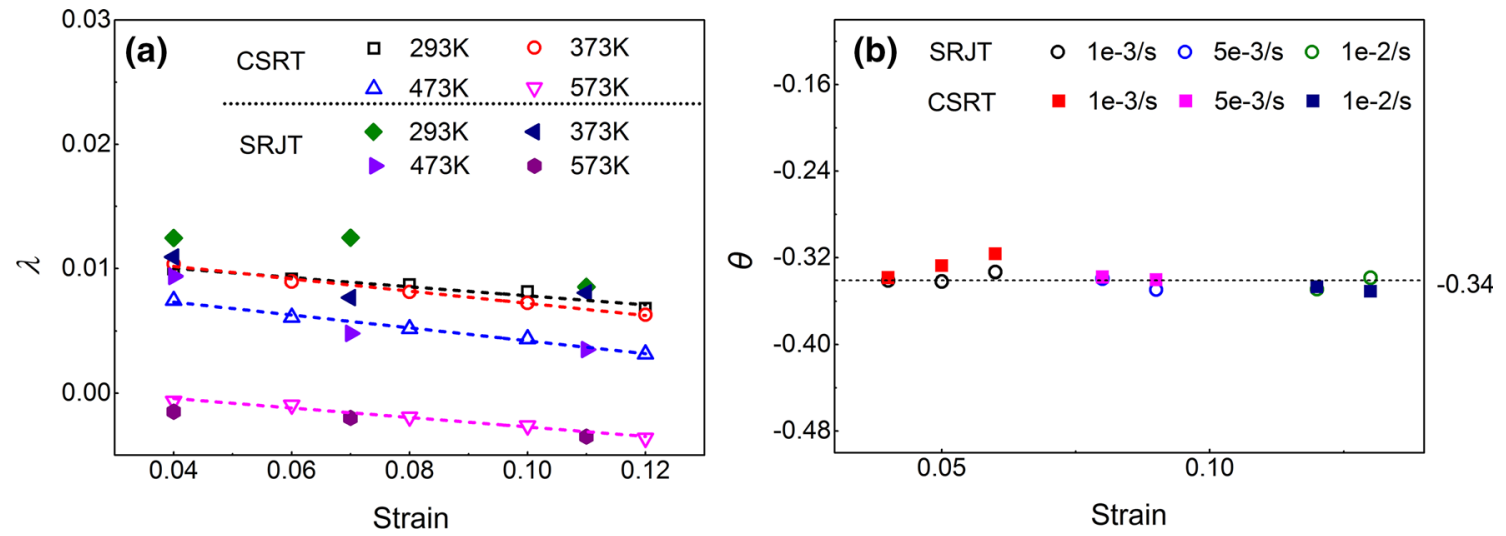

Fig. 6 Comparison between the results of SRJT and CSRT: a SRS indexes; b TS indexes 

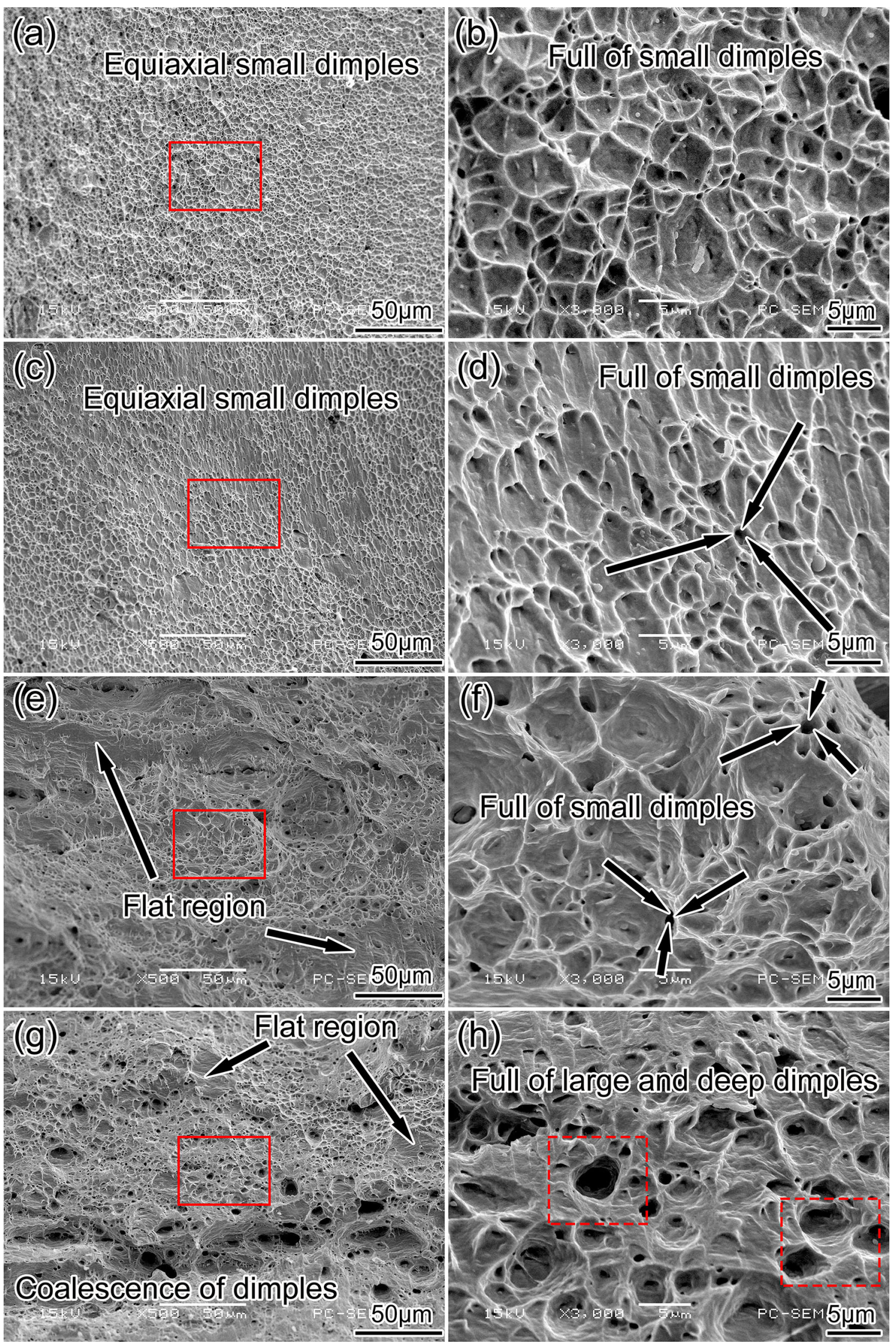
4Fig. 7 Fracture morphologies at different temperatures under the strain rate of $5 \times 10^{-4} / \mathrm{s}$ : a, b $293 \mathrm{~K}$; c, d $373 \mathrm{~K}$; e, f $473 \mathrm{~K}$; g, h $573 \mathrm{~K}$

Ni-based super alloy [26], the coalescence of dimples with the increase in temperature was also found. On the other hand, with the increase in temperature, mixed fracture appearance can be found at 473 and $573 \mathrm{~K}$ as shown in Fig. 7e, g. At $293 \mathrm{~K}$ as shown in Fig. 7a, only equiaxial small dimples can be found on the fracture surface. But with the increase in temperature, the fracture surfaces are mixed with large size dimples and flat regions. Choudhary explained that the flat region provided the evidence of localized transgranular shear fracture at intermediate temperature dominated by DSA [27]. This also agrees with the negative SRS index as discussed in Sects. 3.2 and 3.4. As shown in the fracture morphologies with the magnification of 3000 times, the variation of dimple size and the appearance of flat region can be observed more clearly. Relating to the temperature-dependent stress-strain curves in Fig. 1, the negative TS index in Fig. 6b, as well as the increasing dimple size with temperature in Fig. 7, the temperature softening phenomenon of $316 \mathrm{~L}$ can be depicted. Moreover, relating the appearance of the localized transgranular shear fracture at intermediate temperature in Fig. 7 and the negative SRS index at intermediate temperature in Fig. 4, the DSA can be observed for 316L at intermediate temperature.

On the other hand, in order to analyze the effect of strain rate on fracture morphology of $316 \mathrm{~L}$, fracture morphologies of the minimum strain rate $5 \times 10^{-4} / \mathrm{s}$ and the maximum strain rate $1 \times 10^{-2} / \mathrm{s}$ at $573 \mathrm{~K}$ are compared in Fig. 8. The coalescence of dimples and localized transgranular shear can be found at both strain rates in Fig. 8a, c. Substantial differences of fracture surfaces at different strain rates cannot be observed, which indicates that the effect of strain rate on fracture morphology is weaker than that of temperature. It also agrees with the weaker dependence of stress-strain curves on strain rate as discussed above.

\section{Constitutive Model for 316L at Room and Intermediate Temperatures}

Different constitutive models were proposed to describe the SH, SRS and TS behavior as listed in Table 1. Each model has its specialty and applicable scope. For example, Arrhenius model is based on the thermally activated theory and has significant physical meaning [28, 29], but SRS, TS and SH behaviors are not clearly presented in this model. Moreover, the artificial neural network method is based on artificial intelligence technology as a black box, which cannot visually represent SRS and TS behaviors. Among them, J-C model clearly presents the SH, SRS and TS behaviors, and it is helpful to understand the SH, SRS and TS behaviors of the concerned material. Therefore, in this study, J-C model was applied to present the SRS and TS behaviors of $316 \mathrm{~L}$.

\subsection{Classical Johnson-Cook Model}

Johnson and Cook [11] proposed the classical constitutive model to describe the flow behavior of materials. Since the J-C model can relate stress-strain curve with strain rate and temperature with a clear phenomenological equation, it is widely applied in researches of SRS and TS for various metallic materials. The classical $\mathrm{J}-\mathrm{C}$ model is expressed as Eq. (7), taking SH, SRS and TS independently into consideration.

$\sigma=\left(A+B \varepsilon^{n}\right)\left(1+C \ln \dot{\varepsilon}^{*}\right)\left(1-T^{*^{m}}\right)$,

where $\sigma(\mathrm{MPa})$ and $\varepsilon$ are the flow stress and the equivalent plastic strain, respectively; $A(\mathrm{MPa}), B(\mathrm{MPa})$ are material constants; $C$ is the coefficient of SRS; $n$ and $m$ are strain hardening index and thermal softening index, respectively. In addition, the relative strain rate $\dot{\varepsilon}^{*}$ can be expressed as Eq. (8).

$\dot{\varepsilon}^{*}=\dot{\varepsilon} / \dot{\varepsilon}_{0}$,

where $\dot{\varepsilon}\left(\mathrm{s}^{-1}\right)$ and $\dot{\varepsilon}_{0}\left(\mathrm{~s}^{-1}\right)$ are the experimental strain rate and the reference strain rate.

And the relative temperature can be expressed as:

$T^{*}=\left(T-T_{\text {ref }}\right) /\left(T_{\mathrm{m}}-T_{\text {ref }}\right)$,

where $T(\mathrm{~K}), T_{\mathrm{m}}(\mathrm{K})$ and $T_{\text {ref }}(\mathrm{K})$ are the experimental temperature, the material melting temperature and the reference temperature, respectively.

The choosing basis of the reference temperature and the reference strain rate is to construct the $\mathrm{J}-\mathrm{C}$ model conveniently. Lots of studies $[30,35]$ chose the minimums as the reference temperature and the reference strain rate. Therefore, in this study, the reference strain rate is selected as $5 \times 10^{-4} / \mathrm{s}$, and the reference temperature is selected as $293 \mathrm{~K}$.

\subsubsection{Determination of Parameters $A, B$ and $n$}

At the reference temperature and the reference strain rate, the equation can be simplified as:

$\sigma=A+B \varepsilon^{n}$.

According to Eq. (10), the value of $A$ (MPa) is considered as the yield strength of $316 \mathrm{~L}$, and the values of $B$ 

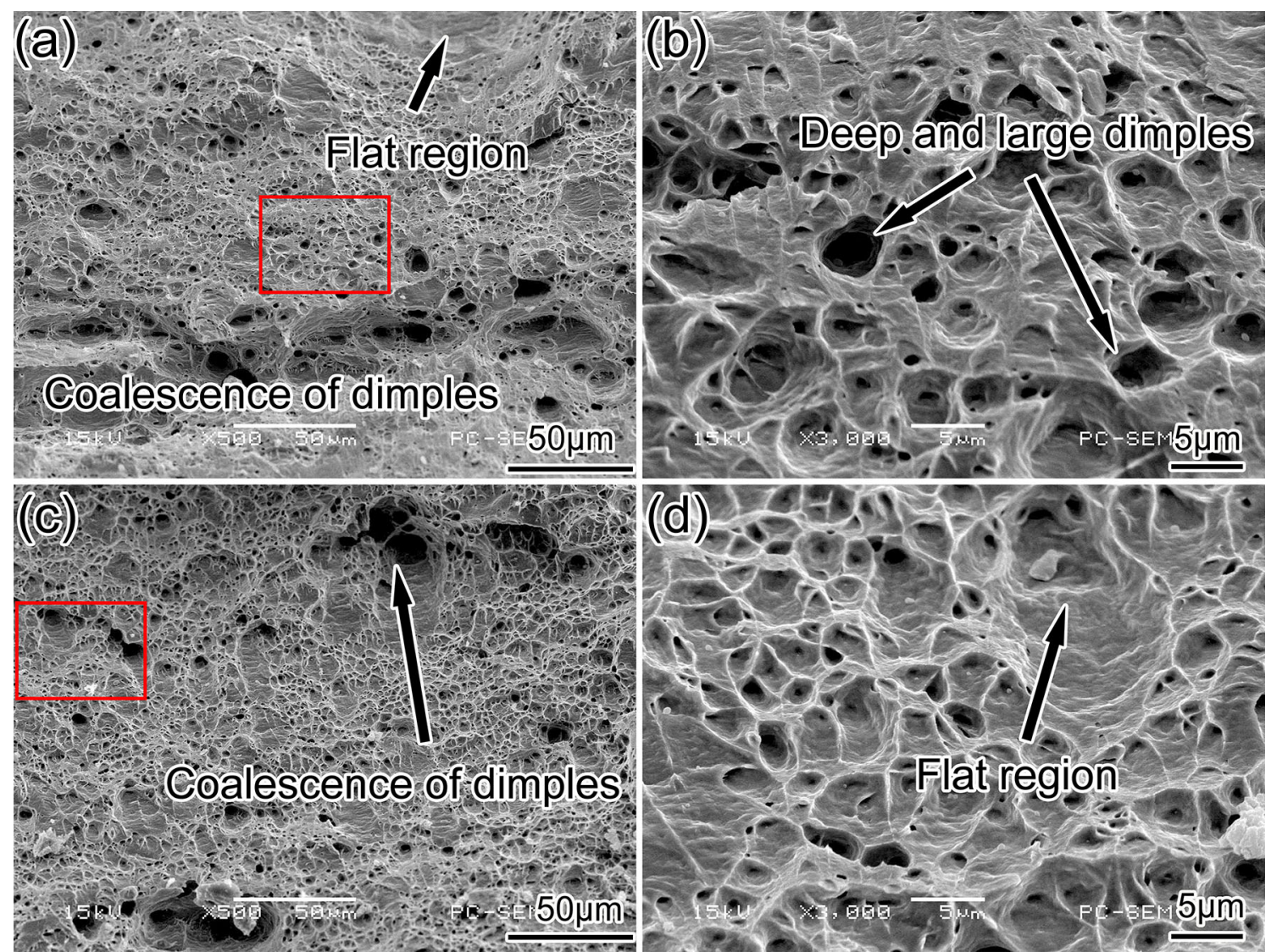

Fig. 8 Fracture morphologies at different strain rates under $573 \mathrm{~K}$ : a, b $5 \times 10^{-4} / \mathrm{s} ; \mathbf{c}, \mathbf{d} 1 \times 10^{-2} / \mathrm{s}$

Table 1 Characteristics of different constitutive models

\begin{tabular}{lll}
\hline & Model equation & Number of material constants \\
\hline Arrhenius [28, 29] & $\dot{\varepsilon}=A[\sinh (\alpha \sigma)]^{n} \exp (-Q / R T)$ & 20 (with 4th polynomial) \\
Johnson-Cook [11, 30] & $\sigma=\left(A+B \varepsilon^{n}\right)\left(1+C \ln \dot{\varepsilon}^{*}\right)\left(1-T^{* m}\right)$ & 5 \\
Zerilli-Armstrong [31, 32] & $\sigma=\left(C_{1}+C_{2} \varepsilon^{n}\right) \exp \left\{\left(C_{3}+C_{4} \varepsilon\right) T^{*}+\left(C_{5}+C_{6} T^{*}\right) \ln \dot{\varepsilon}^{*}\right\}$ & 6 \\
Neural network [33, 34] & Artificial intelligence technology & - \\
\hline
\end{tabular}

(MPa) and $n$ can be calculated by the nonlinear fitting of stress-strain curve, which are illustrated in Fig. 9a.

Then, at the reference temperature, the classical model can be simplified as:

$\sigma=\left(A+B \varepsilon^{n}\right)\left(1+C \ln \dot{\varepsilon}^{*}\right)$.

\subsubsection{Determination of Parameters $C$ and $m$}

Figure $9 \mathrm{~b}$ gives the relationship of $\sigma /\left(A+B \varepsilon^{n}\right)-\ln \dot{\varepsilon}^{*}$, and the value of $C$ can be obtained from the slope of $\sigma /\left(A+B \varepsilon^{n}\right)-\ln \dot{\varepsilon}^{*}$.

Similarly, under the reference strain rate, the classical model can be simplified as Eq. (12).
$\sigma=\left(A+B \varepsilon^{n}\right)\left(1-T^{* m}\right)$.

Figure $9 \mathrm{~b}$ gives the relationship of $\sigma /\left(A+B \varepsilon^{n}\right)-T^{*}$, and the value of $m$ can be obtained from the nonlinear fitting with Eq. (12). The parameter values of the classical $\mathrm{J}-\mathrm{C}$ model are summarized in Table 2.

\subsection{Improved Johnson-Cook model}

Although the $\mathrm{J}-\mathrm{C}$ model is clear to understand $\mathrm{SH}$, SRS, TS behaviors of the concerned material, the coupling effects of SH, SRS, TS are neglected in the classical model. Therefore, many studies improved the $\mathrm{J}-\mathrm{C}$ model by different methods, as listed in Table 3. 

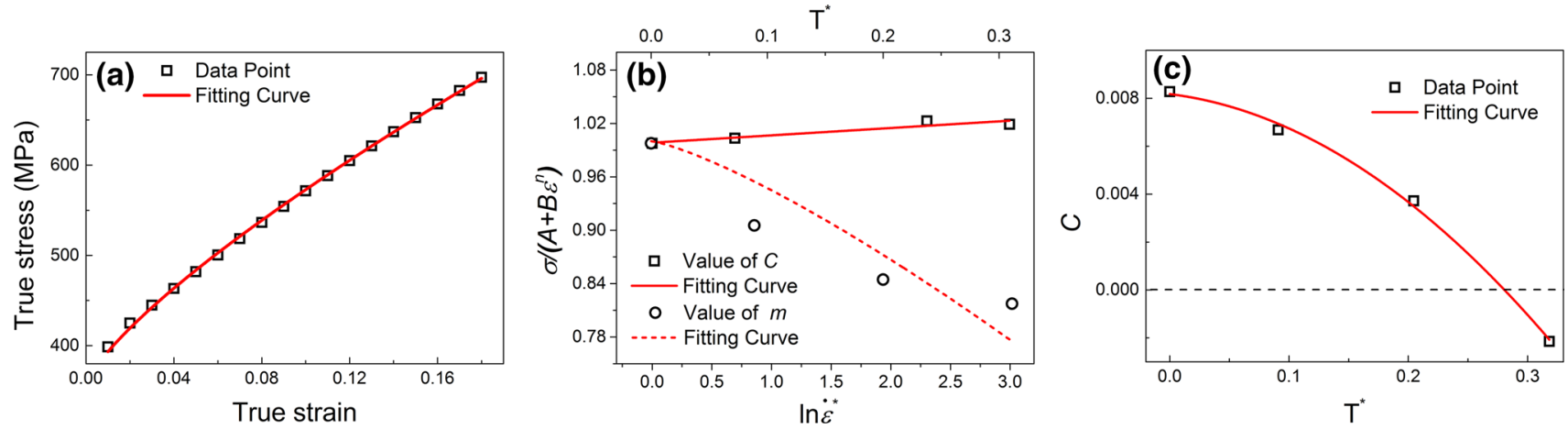

Fig. 9 Calculating procedure of material parameters: a, b material parameters in the classical model; $\mathbf{c}$ variation of $C$ with the relative temperature

Table 2 Parameters of classical J-C model

\begin{tabular}{lllll}
\hline$A(\mathrm{MPa})$ & $B(\mathrm{MPa})$ & $n$ & $C$ & $m$ \\
\hline 356.09 & 1264.35 & 0.77 & 0.008 & 1.28 \\
\hline
\end{tabular}

According to SRS indexes of CSRT and SRJT as shown in Figs. 2, 4 and 6a, the SRS index of 316L is temperature dependent, and the SRS of $316 \mathrm{~L}$ changes from strain rate hardening to strain rate softening with the increase in temperature. On the contrast, in the classical $\mathrm{J}-\mathrm{C}$ model, the SRS parameter $C$ is calculated only by the results at the reference temperature, without considering the effect of temperature on SRS. It suggests that the classical J-C model is not suitable for the experimental results of $316 \mathrm{~L}$. Lin et al. [16] improved J-C model by considering the coupled effects of strain rate and deformation temperature on flow stress, but the modified J-C model cannot illustrate the effect of temperature on SRS index. In order to suitably describe the SRS and TS behaviors of 316L by J-C model, the classical model needs to be improved by considering the temperature dependence of the SRS parameter $C$.

\subsubsection{Determination of Parameters $C_{1}, C_{2}$ and $C_{3}$}

The SRS parameters $C$ at different temperatures are determined by Eq. (11) and shown in Fig. 9c. According to Fig. 9c, the parameter $C$ decreases gradually with the increase in temperature. And the result is consistent with the analyses of the SRS index in Figs. 2, 4 and 6a. Considering the effect of temperature, the quadratic polynomial fitting is precise enough to describe the temperature-dependent SRS behavior, as shown in Fig. 9c, and the relationship between $C$ and $T^{*}$ is expressed as Eq. 13:

$C=C_{1}+C_{2} T^{*}+C_{3} T^{* 2}$,

where $C_{1}, C_{2}$ and $C_{3}$ are material constants, which can present the influence of temperature on SRS.

\subsubsection{Determination of Parameters $D_{1}, D_{2}$ and $D_{3}$}

Based on the fitting curve in Fig. 9c, there are some deviations to describe the relationship of $\sigma /\left(A+B \varepsilon^{n}\right)-T^{*}$ by Eq. 12 . In order to improve the representation of the improved $\mathrm{J}-\mathrm{C}$ model, the quadratic polynomial was utilized to describe the relationship of $\sigma /\left(A+B \varepsilon^{n}\right)-T^{*}$, which is expressed as follows.

$\sigma /\left(A+B \varepsilon^{n}\right)=D_{1}+D_{2} T^{*}+D_{3} T^{* 2}$,

where $D_{1}, D_{2}$ and $D_{3}$ are material constants, which can be considered as the thermal softening parameters.

With the analysis above, the improved $\mathrm{J}-\mathrm{C}$ model can be described as:

$$
\begin{aligned}
\sigma= & \left(A+B \varepsilon^{n}\right)\left[1+\left(C_{1}+C_{2} T^{*}+C_{3} T^{* 2}\right) \ln \dot{\varepsilon}^{*}\right]\left(D_{1}+D_{2} T^{*}\right. \\
& \left.+D_{3} T^{* 2}\right) .
\end{aligned}
$$

The parameters of the improved $\mathrm{J}-\mathrm{C}$ model are listed in Table 4.
Table 3 Development of Johnson-Cook model

\begin{tabular}{lll}
\hline Researchers & Materials & Improvement \\
\hline Lin et al. [16] & Alloy steel & Considering the coupled effects of strain rate and temperature \\
Li et al. [17] & & \\
He et al. [36] & & \\
Zhao et al. [37] & Fe-Cr alloy & Considering strain rate softening \\
Wang et al. [38] & Inconel 718 & Relating parameter $C$ with strain rate and temperature by sine function \\
\hline
\end{tabular}


Table 4 Parameters of the improved $\mathrm{J}-\mathrm{C}$ model

\begin{tabular}{lllllllll}
\hline$A(\mathrm{MPa})$ & $B(\mathrm{MPa})$ & $n$ & $C_{1}$ & $C_{2}$ & $C_{3}$ & $D_{1}$ & $D_{2}$ & $D_{3}$ \\
\hline 356.09 & 1264.35 & 0.77 & 0.008 & 0.006 & 0.08 & 0.996 & -1.14 & 1.83 \\
\hline
\end{tabular}

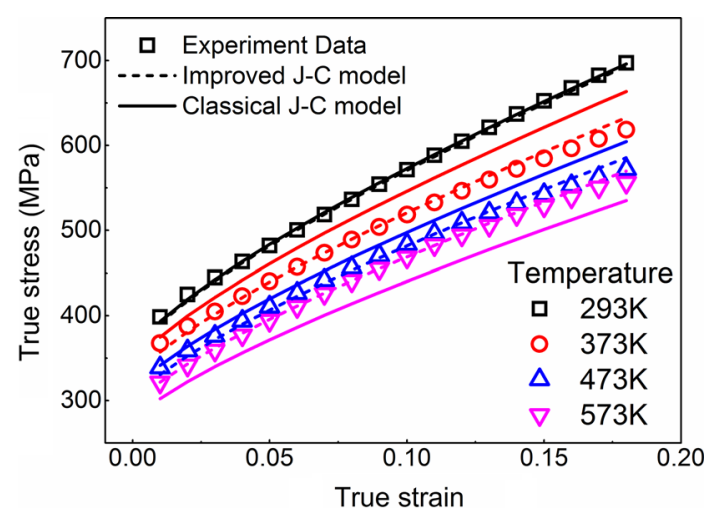

Fig. 10 Comparison of predicted curves between classical $\mathrm{J}-\mathrm{C}$ model and improved $\mathrm{J}-\mathrm{C}$ model (take strain rate $=5 \times 10^{-4} / \mathrm{s}$ as an example)

\subsubsection{Comparison Between Classical and Improved J-C Model}

According to the parameters in Tables 2 and 4, the stressstrain curves under different temperatures and strain rates can be predicted by the classical and the improved $\mathrm{J}-\mathrm{C}$ model, respectively. The predicted curves obtained by the classical $\mathrm{J}-\mathrm{C}$ model and the improved $\mathrm{J}-\mathrm{C}$ model are compared with experimental curves as shown in Fig. 10, which takes strain rate $=5 \times 10^{-4} / \mathrm{s}$ as an example, and results at other strain rates are similar. As shown in Fig. 10, the deviation of prediction curves corresponding to the classical J-C model with experimental data increases with temperature. But the prediction curves of the improved $\mathrm{J}-\mathrm{C}$ model can give better results.

In order to quantitatively analyze the errors between prediction data and the experimental data, some statistical parameters (correlation coefficient and mean error) are introduced. The mathematical expressions of correlation coefficient and mean error are as follows:

$$
\begin{aligned}
& R^{\prime}=\sum_{i=1}^{i=N}\left(\sigma_{\mathrm{E}}^{i}-\bar{\sigma}_{\mathrm{E}}\right)\left(\sigma_{\mathrm{P}}^{i}-\bar{\sigma}_{\mathrm{P}}\right) / \\
& \sqrt{\sum_{i=1}^{i=N}\left(\sigma_{\mathrm{E}}^{i}-\bar{\sigma}_{\mathrm{E}}\right)^{2} \sum_{i=1}^{i=N}\left(\sigma_{\mathrm{P}}^{i}-\bar{\sigma}_{\mathrm{P}}\right)}, \\
& \bar{\Delta}=\frac{1}{N} \sum_{i=1}^{i=N}\left|\left(\sigma_{\mathrm{E}}-\sigma_{\mathrm{P}}\right) / \sigma_{\mathrm{E}}\right| \times 100,
\end{aligned}
$$

where $\sigma_{\mathrm{E}}$ and $\sigma_{\mathrm{P}}$ are the experimental data and the prediction data, $\bar{\sigma}_{\mathrm{E}}$ and $\bar{\sigma}_{\mathrm{P}}$ are the average experimental data and prediction data and $N$ is the number of data.

Correlation coefficients and mean errors of the classical $\mathrm{J}-\mathrm{C}$ model and the improved $\mathrm{J}-\mathrm{C}$ model are calculated at different temperatures. As shown in Fig. 11 at $293 \mathrm{~K}$, the mean error of the classical $\mathrm{J}-\mathrm{C}$ model is the minimum and the correlation coefficient is closed to 1.0. The reason is that the reference temperature is taken as the minimum value in the classical model. However, with the increase in temperature, the mean error increases, while the correlation coefficient is deviating from 1.0. The classical JohnsonCook model requires fewer material constants and also few experiments to evaluate these constants, and it assumes that thermal softening, strain rate hardening and strain hardening are three independent phenomena and can be isolated from each other. Therefore, the classical Johnson-Cook model cannot adequately describe the high-temperature flow behavior with coupled effects of temperature, strain rate and strain. The strain rate sensitivity parameter $C$ of the classical $\mathrm{J}-\mathrm{C}$ model is temperature independent, which disagrees with the temperature-dependent SRS behavior of 316L austenitic stainless steel in Figs. 1b, 2 and $6 a$.
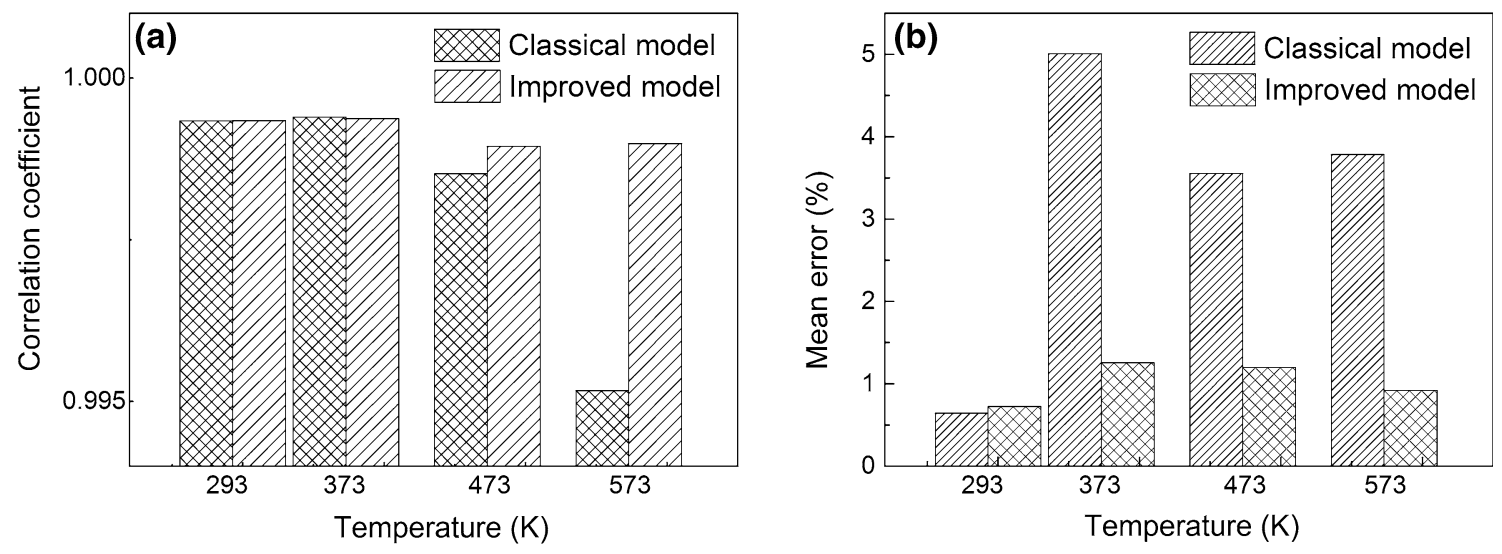

Fig. 11 Comparison of statistical parameters between classical J-C model and improved $\mathrm{J}-\mathrm{C}$ model at different temperatures 
Therefore, the prediction accuracy of the $\mathrm{J}-\mathrm{C}$ model decreases with temperature as shown in Fig. 11.

Since the improved J-C model suitably considers the temperature-dependent SRS behavior with Eq. (13), correlation coefficients and mean errors of the improved $\mathrm{J}-\mathrm{C}$ model hardly vary with temperature. Above all, comparing statistical parameters of two constitutive models, the improved $\mathrm{J}-\mathrm{C}$ model considering the temperature-dependent SRS behavior can describe the SRS and TS of $316 \mathrm{~L}$ with higher accuracy.

\section{Conclusions}

The SRS and TS of $316 \mathrm{~L}$ are systematically studied by CSRT and SRJT at $T=293-573 \mathrm{~K}$, and $\dot{\varepsilon}=5 \times 10^{-4}$ / $\mathrm{s}^{-1} \times 10^{-2} / \mathrm{s}$ focusing on the temperature-dependent SRS behavior. Some conclusions can be obtained as follows:

1. According to the comparison of CSRT and SRJT, TS indexes and SRS indexes obtained by both methods are in good agreement. It proves that the SRJT method is an appropriate option to study the SRS and TS of $316 \mathrm{~L}$.

2. Based on the results of CSRT and SRJT, the SRS index of $316 \mathrm{~L}$ changes from positive to negative with the increase in temperature, which reveals the temperature-dependent SRS behavior of $316 \mathrm{~L}$, and is related to the appearance of DSA.

3. Considering the temperature-dependent SRS behavior, an improved J-C model was proposed, which can describe the SRS and TS of $316 \mathrm{~L}$ tensile behavior properly.

Acknowledgements This project is financially supported by the National Natural Science Foundation of China (Grant No. 51505041) and the Natural Science Foundation of the Jiangsu Higher Education Institutions of China (Grant No. 16KJB460002).

\section{References}

[1] Y.C. Lin, M. He, M. Zhou, D.X. Wen, J. Chen, J. Mater. Eng. Perform. 24, 3527 (2015)

[2] Y. Yan, W.P. Deng, Z.F. Gao, Acta Metall. Sin. (Engl. Lett.) 29, $163(2013)$

[3] J. Peng, C.Y. Zhou, Q. Dai, X.H. He, Mater. Des. 50, 968 (2013)
[4] X.F. Li, J. Chen, L.Y. Ye, Acta Metall. Sin. (Engl. Lett.) 26, 657 (2013)

[5] H.K. Yang, Y.Z. Tian, Z.J. Zhang, Z.F. Zhang, Mater. Sci. Eng. A 655, 251 (2016)

[6] P. Verma, S. Rao, P. Chellapandi, Mater. Sci. Eng. A 621, 39 (2015)

[7] X.D. Bian, F.P. Yuan, X.L. Wu, Mater. Sci. Eng. A 696, 220 (2017)

[8] R. Kasadaa, S. Konishia, D. Hamaguchi, Fusion. Eng. Des. 109, 1507 (2016)

[9] S.R. Bodner, Y. Partom, J. Appl. Mech. 42, 385 (1975)

[10] H.S. Chen, W.X. Wang, H.H. Nie, Y.L. Li, Q.C. Wu, P. Zhang, Acta Metall. Sin. (Engl. Lett.) 28, 1214 (2015)

[11] G.R. Johnson, W.H. Cook, Eng. Fract. Mech. 21, 31 (1985)

[12] F.J. Zerilli, R.W. Armstrong, J. Appl. Phys. 61, 1816 (1987)

[13] Z.B. Huang, M. Wan, H. Wu, J. Plast. Eng. 20, 89 (2013)

[14] Y.C. Lin, X.M. Chen, Mater. Des. 32, 1733 (2011)

[15] Y.C. Lin, Q.F. Li, Y.C. Xia, Mater. Sci. Eng. A 534, 654 (2012)

[16] Y.C. Lin, X.M. Chen, G. Liu, Mater. Sci. Eng. A 527, 6980 (2010)

[17] H.Y. Li, Y.H. Li, X.F. Wang, Mater. Des. 49, 493 (2013)

[18] W.C. Jiang, J.M. Gong, S.T. Tu, Mater. Des. 31, 2157 (2010)

[19] W.C. Jiang, B. Yang, X.W. Guan, Y. Luo, Acta Metall. Sin. (Engl. Lett.) 26, 241 (2013)

[20] P. Ferro, A. Fabrizi, F. Bonollo, Acta Metall. Sin. (Engl. Lett.) 29, 859 (2016)

[21] GB/T 228.1-2010

[22] W.A. Backoken, I.R. Turner, D.H. Avery, J. Mater. Sci. 6, 1061 (1971)

[23] K.W. Qian, J. Fuzhou. Univ. 16, 57 (1988)

[24] E.I. Samuel, B.K. Choudhary, K.B.S. Rao, Scr. Mater. 46, 507 (2002)

[25] Y.Q. Song, Z.P. Guan, Z. Li, Sci. China (Ser. E) 37, 1363 (2007)

[26] Y.C. Lin, J. Deng, Y.Q. Jiang, Mater. Des. 55, 949 (2014)

[27] B.K. Choudhary, Metall. Mater. Trans. A 44, 4979 (2013)

[28] J. Yan, Q.L. Pan, A.D. Li, W.B. Song, Trans. Nonferrous Met. Soc. China 27, 638 (2017)

[29] Y.H. Zhang, W. Zhang, J.L. Gao, Z.M. Yuan, W.G. Bu, Acta Metall. Sin. (Engl. Lett.) 30, 1040 (2017)

[30] F. Ducobu, E. Rivière-Lorphèvre, E. Filippi, Int. J. Mech. Sci. 122, 143 (2017)

[31] T. Mirzaiea, H. Mirzadeha, J.M. Cabrera, Mech. Mater. 94, 38 (2016)

[32] D.Y. Li, Z.W. Zhu, S.N. Xiao, G.H. Zhang, Y.S. Lu, Mater. Sci. Eng. A 707, 459 (2017)

[33] D.D. Chen, Y.C. Lin, Y. Zhou, M.S. Chen, D.X. Wen, J. Alloys Compd. 708, 938 (2017)

[34] Y.C. Lin, Y.J. Liang, M.S. Chen, X.M. Chen, Appl. Phys. A 123, 68 (2017)

[35] L. Gambirasio, E. Rizzi, Comput. Mater. Sci. 113, 231 (2016)

[36] A. He, G.L. Xie, H.L. Zhang, X.T. Wang, Mater. Des. 52, 677 (2013)

[37] Y.H. Zhao, J. Sun, J.F. Li, Y.Q. Yan, P. Wang, J. Alloys Compd. 723, 179 (2017)

[38] X.Y. Wang, C.Z. Huang, B. Zou, H.L. Liu, H.T. Zhu, J. Wang, Mater. Sci. Eng. A 580, 385 (2013) 